\title{
The Pro-oxidant Activity and Composition of Polar Compound Fractions in Used Deep-frying Camellia Seed Oil
}

\author{
Jinying Wang ${ }^{1}$, Haiyan Zhong ${ }^{2, *}$, Qizhi Long ${ }^{2}$, Paul D Prenzler ${ }^{3}$ \\ ${ }^{1}$ College of Agriculture and Animal Husbandry, Qinghai University, Xining, P R China \\ ${ }^{2}$ College of Food Science and Engineering, Central South University of Forestry and Technology, Changsha, P R China \\ ${ }^{3}$ School of Agricultural and Wine Sciences, EH Graham Centre for Agricultural Innovation, Charles Sturt University, \\ Wagga Wagga 2678, Australia \\ *Corresponding author: zhonghaiyan631210@126.com
}

\begin{abstract}
We aimed to investigate the pro-oxidant activity of polar compounds in deep-frying camellia seed oil (CO) using the accelerated oxidation test, and also to analyze the fractions and distribution of its polar compounds using high performance size exclusion chromatography (HPSEC). Our results indicated that the addition of previously isolated polar compounds to four types of CO (purified refined, unpurified refined, purified crude, and unpurified crude) reduced the oxidation induction time, which suggested that these compounds were actively prooxidant. This result is critical for the safe storage of refined oil, and for the quality monitoring of frying oil. Our HPSEC analysis showed that used CO contained polar compounds (TGO, TGD, ox-TGM, DG, and FFA). After 40 $\mathrm{h}$ of deep frying, the polymerization products (TGO and TGD), oxidative products (ox-TGM), and hydrolytic products (DG and FFA) comprised 54.63\%, 33.54\%, and 15.14\% of the total polar content, respectively, suggesting that the hydrolysis reaction during the deep frying was weak compared to thermal oxidation polymerization. Based on this result, in combination with our measurement of the concentration of total polar compounds (TPC, 27\%) and triacylglycerol polymers (TGP, 10-16\%), we postulate that the frying life of CO is $32 \mathrm{~h}$.
\end{abstract}

Keywords: camellia seed oil, deep frying, polar compounds, pro-oxidant activity

Cite This Article: Jinying Wang, Haiyan Zhong, Qizhi Long, and Paul D Prenzler, "The Pro-oxidant Activity and Composition of Polar Compound Fractions in Used Deep-frying Camellia Seed Oil." Journal of Food and Nutrition Research, vol. 6, no. 2 (2018): 124-129. doi: 10.12691/jfnr-6-2-9.

\section{Introduction}

Deep fat frying is defined as the process of cooking foods by immersing them in edible fat or oil at a temperature above the boiling point of water, usually $150-200^{\circ} \mathrm{C}$ [1]. The speed, palatability, and considerable economic benefit of deep frying make this cooking method one of the most popular in homing kitchens, fastfood restaurants and instant noodles industry [2]. During deep-frying, both heat and mass are simultaneously transferred. Heat is transferred from the frying medium to the product surface by convection, and from the surface to the inside by conduction. Moisture, on the other hand, is evaporated and transported from the interior to the surface of the solid by diffusion, and then migrates from the surface through the frying medium [3]. The intimate contact between the food and the oil, described by the popular Blumenthal's Surfactant Theory of Frying, makes frying more efficient than either dry or wet heat [1]. The absorption and adsorption of oil, the surface dehydration of food with the consequent crust formation, the development of surface color and the generation of flavor cumulatively account for the universally desired taste of fried food $[4,5]$.

Polar compounds with higher molecular weights $(\mathrm{Mw})$ than triacylglycerol formed after a series of chemical reactions (polymerization, oxidation and hydrolysis) during deep-frying [6,7]. These polar compounds can be separated via size-exclusion chromatography into 5 groups according to molecular diameter or weight: triacylglycerol oligomers (TGO; Mw 2469), triacylglycerol dimers (TGD; Mw 1728), oxidized triacylglycerol monomers (ox-TGM; Mw 908), diacylglycerols (DG; $M w$ 510), and free fatty acids (FFA; Mw) [8,9,10]. These compounds also have different nutritional effects. For example, Gomes reported that ox-TG and TGP (triacylglycerol polymers: TGO+TGD) in oil polar components had a strong pro-oxidant effect, and that the pro-oxidant activity of ox-TG was much higher than TGP [11]. Furthermore, animal experiments have indicated that polar components have significant biological toxicity, can damage immune-system function [12]. Long-term ingestion of repeatedly heated oil has negative effects on the activity of blood pressure-regulating enzymes, can further aggravate lipid peroxidation, increase blood pressure, and therefore may aggravate various diseases including cardiovascular 
disease and type 2 diabetes [13,14]. To combat this, many countries have enacted laws to stipulate maximum levels of intake of total polar compounds (TPC) in the diet (24-27) [15]. TPC is related to TGP [16]. Some European countries use TGP to evaluate frying oil and set the maximum intake limit at $10-16 \%$ [17]. However, China has not explicitly stipulated a maximum limit of TGO in frying oil.

Camellia seed oil (CO) has been used as a cooking oil in China for 2300 years [18]. It is characterized by the high proportion of oleic acid (74-89\%) [19], and presence of antioxidant compounds such as sterols, triterpenes [20], tocopherol, squalene [21] and phenolics [22,23]. In this study, we aimed to I nvestigate the pro-oxidant activity of polar compounds in CO used for deep-frying with an accelerated oxidation test, and then to analyze the components and distribution of polar compounds in the CO using high performance size exclusion chromatography (HPSEC).

\section{Materials and Methods}

\subsection{Materials}

The FFA, PV and $p-\mathrm{AV}$ of fresh $\mathrm{CO}$ were $0.22 \%$, $3.13 \mathrm{mmol} / \mathrm{kg}$ and 4.91 respectively. The fresh CO contained (per $100 \mathrm{~g}$ ): 8.42 g palmitic acid, 1.73 g stearic acid, $79.91 \mathrm{~g}$ oleic acid, $8 \mathrm{~g}$ linoleic acid, and $0.44 \mathrm{~g}$ linolenic acid. All chemicals and solvents used were either analytical grade or high performance liquid chromatography (HPLC) grade.

\subsection{Purification of $\mathrm{CO}$}

We purified the CO using the methods described by Gong [24,25] and Kleiner-Shuhler [26] with some slight modifications. We used a glass column packed with four fillers to wipe off the micro-compounds in the CO. We used a $60 \mathrm{~cm} \times 4 \mathrm{~cm}$ column packed with $75 \mathrm{~g}$ of silica gel, 70-230 mesh; 12.6 g charcoal: celite (2:1, w/w); 37.5 g sucrose powder: celite (2:1, w/w); and 75 g of silica gel. The fillers, immersed in $n$-hexane, were introduced in the glass column using the wet-packing method and compacted by a three-way valve connection double ball. We resolved a $250 \mathrm{~g}$ oil sample in isometric n-hexane and eluted it until the fractions were free of oil stains on filter paper. The eluents were removed with rotary evaporator at $50^{\circ} \mathrm{C}$. The resulting purified oil was stored at $-20^{\circ} \mathrm{C}$.

\subsection{Frying Protocol}

Frying experiments were carried out using a domestic deep-fat electric fryer (HY-81, GangYang electromechanical equipment company) equipped with a removable stainless steel wire basket and a thermostat. Fresh potatoes were peeled quickly, cut into chips (approximately 40-50 × 10 $\times 10 \mathrm{~mm}$ ), rinsed, and drained thoroughly. Before frying the first batch of potatoes, we heated the fresh CO to $185 \pm 5^{\circ} \mathrm{C}$ for 30 min to condition it. Each $125 \mathrm{~g}$ batch of potato chips was fried for $4 \mathrm{~min}$ at $11 \mathrm{~min}$ intervals for a period of 6 hours per day, a total of 24 batches fried per day for 7 consecutive days. Aliquots of $50 \mathrm{~g}$ used oil were transferred into amber bottles at 2 hours intervals, cooled to room temperature, and stored at $-20^{\circ} \mathrm{C}$ for further analysis. Deep frying experiments were carried out in triplicate.

\subsection{Determination of Carbonyl Value}

The carbonyl value (CV) of the used oil was determined according to Chinese National Standards [27].

\subsection{Separation of the Polar Compounds}

The TPCs of the used oil were determined using classical column chromatography [28,29,30,31] with some modifications. We dissolved $1.00 \mathrm{~g}$ oil samples in $10 \mathrm{~mL}$ solvent (light petroleum/diethyl ether, 87/13, v/v), and then introduced this solution into a glass column $(20 \times$ $400 \mathrm{~mm}$ ) filled with a slurry of silica gel (70-230 mesh). We used $150 \mathrm{~mL}$ of solvent 1 (n-hexane/diethyl ether, $87 / 13, \mathrm{v} / \mathrm{v}$ ) and $150 \mathrm{~mL}$ of solvent 2 (diethyl ether, b.p. $40-60^{\circ} \mathrm{C}$ ) to elute the non-polar and polar compounds, respectively. A dropping funnel was used to maintain a flow rate of about $2.5 \mathrm{~mL} / \mathrm{min}$. The solvents were evaporated, and the contents of the non-polar and polar fractions were determined gravimetrically. Efficiency of the separation was checked by thin layer chromatography [28]. The mass fractions of non-polar compounds $\left(\mathrm{W}_{N P C}\right)$ and the polar compounds $\left(\mathrm{W}_{P C}\right)$ were given by the following formulas:

$$
\begin{gathered}
W_{N P C}=\frac{M_{N P C}}{M} \times 100 \% \\
W_{P C}=\frac{M_{P C}}{M} \times 100 \%
\end{gathered}
$$

Where $M_{\mathrm{NPC}}$ is the mass of the non-polar fraction (g), $M_{\mathrm{PC}}$ is the mass of the polar fraction (g), and $M$ is the mass of the test portion added to the column (g).

\subsection{Determination of the Pro-oxidation Activity of the Polar Compounds}

We prepared a stock solution of TPCs were separated from aliquots of CO fried for 10, 20, 30, and $40 \mathrm{~h}$ using n-hexane as described in section 2.5. Appropriate stock amounts of this solution were added to Rancimat reaction vessels. Once the solvent was completely removed under nitrogen, purified and unpurified CO were added to each vessel to make exactly $5 \mathrm{~g}$. The concentrations of polar compounds in purified and unpurified oil samples obtained were 2, 4, 6 and 8\%. We used one vessel containing only purified oil as a negative control. We then used the 842 Rancimat apparatus (Metrohm, Switzerland) to perform accelerated oxidation tests on the reaction vessels. Each sample was tested three times to determine oxidation stability; we also computed the means and standard deviations of the results. For the purified refined and crude $\mathrm{CO}$, the temperature was $85^{\circ} \mathrm{C}$ with an airflow of $20 \mathrm{~L} / \mathrm{h}$, while for the unpurified refined and crude $\mathrm{CO}$, temperature was $105^{\circ} \mathrm{C}$ with an airflow of $20 \mathrm{~L} / \mathrm{h}$. 


\subsection{Quantification and Distribution of Polar Compounds}

We recovered the polar fractions obtained in section 2.5 in $10 \mathrm{~mL}$ tetrahydrofuran, and analyzed them using HPSEC, according to the methods in the literatures [31,32]. We followed the same methods to analyze PTG. We separated polar compounds of different polarity and molecular size using two $100 \AA$ and $500 \AA$ PL gel columns $(30 \times 0.75 \mathrm{~cm}$ I.D. $)$ packed with porous, highly cross-linked polystyrene-divinylbenzene copolymers connected in series (film thickness: $5 \mu \mathrm{m}$ ) (Agilent Technologies, USA). HPLC-grade tetrahydrofuran served as the mobile phase with a flow rate of $1 \mathrm{~mL} / \mathrm{min}$. Sample concentration was $7-12 \mathrm{mg} / \mathrm{mL}$ in tetrahydrofuran. The temperature of the column and the detector was kept at a constant $35^{\circ} \mathrm{C}$ for the whole of the analysis (20 min). Five groups of compounds were separated and quantified: TGO, TGD, ox-TGM, DG, and FFA (most to least abundant). We assumed that all sample compounds were eluted. The composition of the used oil can be calculated as follows:

$$
\begin{aligned}
\mathrm{W}_{\mathrm{TGO}} & =\frac{A_{\mathrm{TGO}}}{\sum A} \times W_{\mathrm{PC}} \\
W_{\mathrm{TGD}} & =\frac{A_{\mathrm{TGD}}}{\sum A} \times W_{\mathrm{PC}} \\
W_{\mathrm{OX}-\mathrm{TGM}} & =\frac{A_{\mathrm{OX}-\mathrm{TGM}}}{\sum A} \times W_{\mathrm{PC}} \\
W_{\mathrm{DG}} & =\frac{A_{\mathrm{DG}}}{\sum A} \times W_{\mathrm{PC}} \\
W_{\mathrm{FFA}} & =\frac{A_{\mathrm{FFA}}}{\sum A} \times W_{\mathrm{PC}}
\end{aligned}
$$

Where $W_{\mathrm{TGO}}, W_{\mathrm{TGD}}, W_{\mathrm{Ox}-\mathrm{TGM}}, W_{\mathrm{DG}}$, and $W_{\mathrm{FFA}}$ are the percentages of TGO, TGD, ox-TGM, DG, and FFA in the used oil; $A_{\mathrm{TGO}}, A_{\mathrm{TGD}}, A_{\mathrm{ox}-\mathrm{TGM}}, A_{\mathrm{DG}}$ and $A_{\mathrm{FFA}}$ are the peak areas of under a specific fraction; $\sum A$ is the sum of all peak areas; and $\mathrm{W}_{\mathrm{PC}}$ is the total percentage of polar compounds in the oil.

\subsection{Statistical Analysis}

We performed all experiments in triplicate, and present mean \pm SD of the results. We used Origin Pro 8.0 and SPSS17.0 to calculate one-way analysis of variance (ANOVA) and regression. Significant differences among different oil aliquots and treatments were determined with LSD multiple range tests. Significant differences $(\mathrm{P}<0.05)$ were identified using LSD procedures.

\section{Results and Discussions}

\subsection{Pro-oxidant Activity of Polar Compounds}

The oxidation induction time (OSI) of the crude CO was 1.98 times that of the refined sample after purification (Table 1). The Rancimat measurements of the purified crude and refined oils showed that as the amounts of polar compounds increased, the oxidation induction times of both oils decreased. This suggested that the polar compounds separated from the used CO acted as prooxidants in the purified refined and crude oils, which was consistent with the results of Kim [33].

Table 1. The Effect of Added Polar Compounds on The Oxidation Induction Time of Purified Refined and Crude CO $(n=3)$

\begin{tabular}{ccc}
\hline \multirow{2}{*}{$\begin{array}{c}\text { Polar Compounds } \\
(\% \text { w/w })\end{array}$} & Refined oil & Crude Oil \\
\cline { 2 - 3 } & $5.06 \pm 0.63 \mathrm{a}$ & $10.03 \pm 0.56 \mathrm{a}$ \\
2 & $4.66 \pm 0.89 \mathrm{~b}$ & $9.85 \pm 0.23 \mathrm{~b}$ \\
4 & $3.37 \pm 0.83 \mathrm{c}$ & $9.38 \pm 0.34 \mathrm{c}$ \\
6 & $2.62 \pm 0.34 \mathrm{~d}$ & $7.45 \pm 0.56 \mathrm{~d}$ \\
8 & $2.19 \pm 0.21 \mathrm{e}$ & $6.63 \pm 0.64 \mathrm{e}$ \\
\hline
\end{tabular}

All the values are mean \pm SD $(n=3)$; Values with different lowercase letters $(\mathrm{a}-\mathrm{d})$ within each column were significantly different $(\mathrm{P}<0.05)$.

Specifically, the OSI for purified oils containing 8\% polar compounds was only $2.19 \pm 0.21 \mathrm{~h}$ for refined and $6.63 \pm 0.64 \mathrm{~h}$ for crude. In contrast, OSI for purified oils without polar compounds was $5.06 \pm 0.63 \mathrm{~h}$ for refined and $10.03 \pm 0.56 \mathrm{~h}$ for crude. Thus, the increase OSI for purified oils with polar compounds is $54 \%$ and $33 \%$ for refined and crude oils, respectively. In all samples, prooxidant activity was significantly increased in samples with polar compounds added $(\mathrm{P}<0.05)$. The OSI of purified oils with $2 \%$ polar compounds was $53 \%$ (for refined) and $32 \%$ (for crude) shorter than the OSI of the purified oils with $0.25 \%$ polar compounds.

To further investigate the pro-oxidant activity of polar compounds, we added progressively greater amounts of polar compounds to unpurified refined and crude CO, following the procedure outlined above. The OSI of unpurified oils decreased $41 \%$ (in refined) and 22\% (in crude) as the concentration of polar compounds concentration added increased from $2 \%$ to $8 \%$ (Table 2). The OSI of unpurified refined and crude CO were greater than those of purified oils $(2.29 \times$ purified refined, $1.59 \times$ purified crude), which might be due to the micro-compounds wiped off by absorption chromatography.

Table 2. The Effect of Added Polar Compounds on The Oxidation Induction Time of Unpurified Refined and Crude CO (n=3)

\begin{tabular}{ccc}
\hline \multirow{2}{*}{$\begin{array}{c}\text { Polar Compounds } \\
(\% \mathrm{w} / \mathrm{w})\end{array}$} & Refined oil & OSI $(\mathrm{h})$ \\
\cline { 2 - 3 } & $12.15 \pm 0.55 \mathrm{a}$ & $16.01 \pm 0.81 \mathrm{a}$ \\
2 & $12.08 \pm 0.22 \mathrm{a}$ & $14.73 \pm 0.56 \mathrm{~b}$ \\
4 & $9.97 \pm 0.78 \mathrm{~b}$ & $14.04 \pm 0.23 \mathrm{c}$ \\
6 & $8.84 \pm 0.34 \mathrm{c}$ & $13.32 \pm 1.05 \mathrm{~d}$ \\
8 & $7.16 \pm 0.45 \mathrm{~d}$ & $12.18 \pm 0.73 \mathrm{e}$ \\
\hline
\end{tabular}

All the values are mean \pm SD $(n=3)$. Values with different lowercase letters $(\mathrm{a}-\mathrm{d})$ within each column are significantly different $(\mathrm{P}<0.05)$.

Our results indicate that OSI in unpurified oil decreased with the addition of larger concentrations of polar compounds, which demonstrated that pro-oxidant activity persist in unpurified oil as well as purified, consistent with the results of Gomes [11].

OSI levels of oils with added polar compounds were significantly greater than OSI levels for oils with no polar compounds, at all polar compound concentrations tested (Table 2). However, there is no statistical difference 
between refined oil without polar compounds and unrefined oil to which the lowest amounts of polar compounds 2\% was added. Gomes [9] suggested that because refined edible oil already contains polar compounds (and other anti-oxidant or pro-oxidant substances) as a result of the refining and hydrogenation process, a small amount of polar compounds (2\%) is balanced, whereas the addition of greater amounts causes the pro-oxidant activity of the polar compounds to prevail. We found that the unpurified $\mathrm{CO}$ exhibited considerable resistance to severe oxidative conditions as compared to the purified oil. In addition, as compared to refined CO, the crude oil exhibited greater oxidation stability. These results may be ascribed to the array of anti-oxidants naturally present in unpurified and crude oils.

\subsection{Quantification and Distribution of Polar Compounds}

In fresh CO, TPC was $1.01 \%$, and was significantly positively correlated with deep-frying time $\left(\mathrm{R}^{2}>0.99\right.$;
Table 3), consistent results from other edible oils [34,35]. During deep-frying, CO TPC increased gradually $(0.85 \% / h)$ to a peak of $35.51 \%$ after $40 \mathrm{~h}$. In China, the maximum limit of TPC in frying oil is $27 \%$; we found that the TPC content was $24.19 \%$ after 24 h frying. Based on these results, we preliminarily estimated the frying life of CO to be 24-26 h.

During deep-frying, the primary polar components formed in CO were TGO, TGD, ox-TGM, DG, and FFA (Table 3). TGD and TGO are polymerized by covalent bonds such as $\mathrm{C}-\mathrm{C}, \mathrm{C}-\mathrm{O}$, and O-O [9]. The Mw of TGP is two or more times greater than the Mw of the TAG monomer [36]. During deep-frying, the TGD content of CO increased 1.42 times in the first $24 \mathrm{~h}$ than it did in the next $20 \mathrm{~h}$; the final concentration of TGD after $40 \mathrm{~h}$ was $12.5 \%$ (Table 3 ). TGO could not be detected in fresh CO, but increased continuously with time and reached $0.52 \%$ after $16 \mathrm{~h}$ of deep-frying (Table 3). During the first $20 \mathrm{~h}$, TGD was generated at a rate lower than that of TGO, but in the following $20 \mathrm{~h}$, the rate of TGO generation was substantially significant, as also found by Kupranycz et al. [37].

Table 3. Percent Concentrations of The Polar Compounds in Used Camellia Seed Frying Oil Over Time

\begin{tabular}{|c|c|c|c|c|c|c|}
\hline \multirow{2}{*}{ Index } & \multicolumn{6}{|c|}{ Time/h } \\
\hline & 0 & 8 & 16 & 24 & 32 & 40 \\
\hline $\mathrm{TPC}$ & $2.21 \pm 0.29 \mathrm{~F}$ & $10.05 \pm 0.18 \mathrm{E}$ & $17.02 \pm 1.02 \mathrm{D}$ & $23.92 \pm 0.29 \mathrm{C}$ & $29.96 \pm 0.31 B$ & $35.51 \pm 0.04 \mathrm{~A}$ \\
\hline TGO & ND & ND & $0.52 \pm 0.03 \mathrm{D}$ & $2.72 \pm 0.08 \mathrm{C}$ & $4.56 \pm 0.07 \mathrm{~B}$ & $6.90 \pm 0.20 \mathrm{~A}$ \\
\hline TGD & $0.19 \pm 0.01 \mathrm{~F}$ & $2.67 \pm 0.06 \mathrm{E}$ & $6.29 \pm 0.51 \mathrm{D}$ & $8.45 \pm 0.13 \mathrm{C}$ & $10.79 \pm 0.06 \mathrm{~B}$ & $12.50 \pm 0.11 \mathrm{~A}$ \\
\hline DG & $1.29 \pm 0.19 \mathrm{C}$ & $2.62 \pm 0.08 \mathrm{~B}$ & $2.98 \pm 0.25 B$ & $3.32 \pm 0.03 B$ & $4.10 \pm 0.26 \mathrm{~A}$ & $4.59 \pm 0.55 \mathrm{~A}$ \\
\hline FFA & $0.7 \pm 0.01 \mathrm{~F}$ & $0.34 \pm 0.06 \mathrm{E}$ & $0.48 \pm 0.11 \mathrm{D}$ & $0.56 \pm 0.14 \mathrm{C}$ & $0.67 \pm 0.15 \mathrm{~B}$ & $0.79 \pm 0.07 \mathrm{~A}$ \\
\hline
\end{tabular}

Note: ND = not detected. All values are mean \pm SD $(n=3)$. Values with different uppercase letters $(A-F)$ within each row are Significantly different $(\mathrm{P}<0.05)$.

TGD and TGO are primary polar components that are known to have some level of biological toxicity [38]. However, Berger et al. suggested that the evaluation of the quality of oils with higher diacylglycerol content (e.g. palm oil and RBD palm oil) at high temperatures was problematic [39]. Therefore, TGP (TGO+TGD) has gradually become known as a more reliable standard for the evaluation of frying oil quality; the maximum limit of TGP in frying oil is $10-16 \%$ [39]. Here, the formation of TGP was positively correlated with the length of the frying time in the used CO $\left(\mathrm{R}^{2}>0.99\right)$. After $24 \mathrm{~h}$, the TGP concentration in the 4 oil samples was $11.17 \%$, within the maximum TGP limit range. After 40 h of deepfrying, TGP in the CO was $19.4 \%$, accounting for $54.62 \%$ of the TPC.

Ox-TGM is a TG monomer containing oxygenation groups (e.g. HOO•, the epoxy group, the ketonic group, and $\mathrm{HO} \bullet$ ) and is formed by TG oxidation during oil processing, frying, and storage [40]. TG monomers may remain in the oil after refinement [41]. As the evaluation of primary oxidative products may provide useful information about the oxidation process, ox-TGM is suitable index with which to evaluate early-stage oil oxidation [42]. Here, we observed that the variation of oxTGM in CO was similar to that of TGD, ox-TGM was generated quickly during the first $20 \mathrm{~h}$, albeit at a slower rate than TGD, with a subsequent slowdown. This result is consistent with that of Farhoosh et al. [43]. Bansal et al. [7] considered that cooling of oil during heat treatment and overnight storage caused the persistent increase of ox-TGM; they postulate that the permeation of oxygen throughout the oil causes the formation of unstable hydroperoxides, which are converted to ox-TGM during the subsequent frying [7]. It has been shown that increases in ox-TGM concentration are proportional to TGD formation [44]. After $40 \mathrm{~h}$ of deep-frying, the concentration of ox-TGM in the used CO was $11.91 \%$.

Quantitative analysis of DG and FFA can detect the distribution of hydrolytic products. Dobarganes et al. [44] and Bansal et al. [7] reported that the concentrations of DG and FFA in used CO were not significantly altered with increased frying time. Nevertheless, our results are similar to those of Farhoosh et al. [43] in that DG and FFA concentrations tended to increase with frying time. After $40 \mathrm{~h}$ of deep-frying, the concentrations of both DG and FFA in the used CO increased, to $4.59 \%(3.59 \% / \mathrm{h})$ and $0.79 \%(0.08 \% / h)$, respectively.

After $40 \mathrm{~h}$ of deep drying, the fractions of polymerization products (TGO and TGD), oxidative products (ox-TGM), and hydrolytic products (DG and FFA) in the used CO were $54.63 \%$, 33.54\% and $15.14 \%$, respectively (Figure 1). Compared with the other polar components, concentration 
of the hydrolytic products (DG and FFA) remained low, suggesting that hydrolysis during frying was weak, and that thermal oxidation was more important.

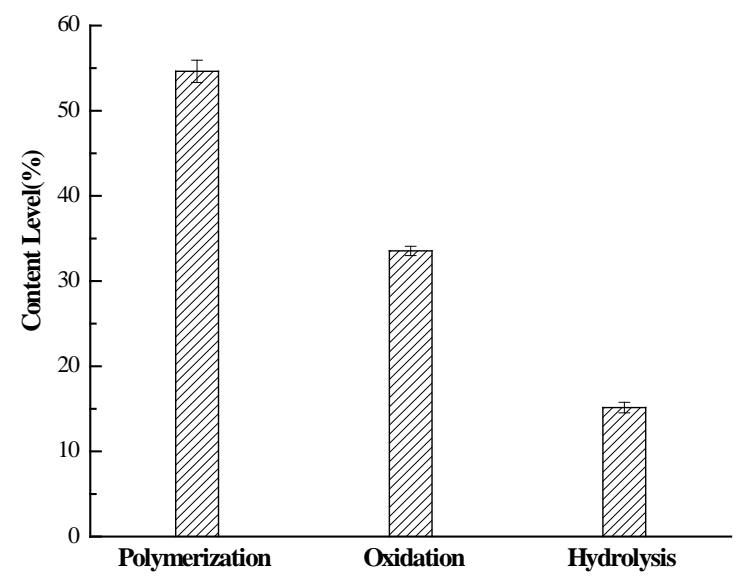

Figure 1. The proportion of polymerization products (TGO + TGD), oxidative products (ox-TGM), and hydrolytic products (DG + FFA) in the total polar compounds of camellia frying oil

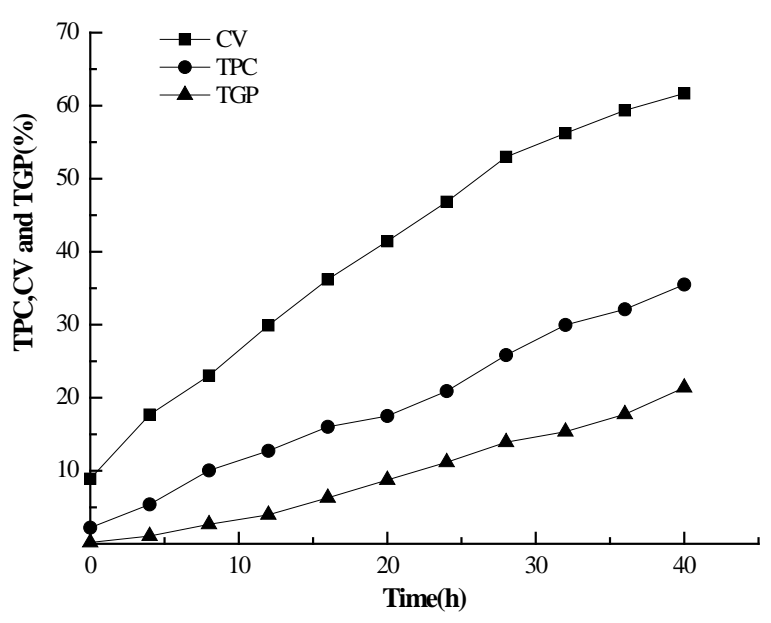

Figure 2. The relationship between total polar compounds (TPC), triacylglycerol polymers (TGP), and carbonyl value (CV) in camellia frying oil

In China, TPC concentration and CV are the main indexes used for evaluating frying oil quality, and the maximum limits are $27 \%$ and $50 \mu \mathrm{mol} / \mathrm{g}$, respectively. The maximum allowed TPC concentration in some European countries is lower (i.e. 24\%). During deep frying, the TPC concentration in our used CO was $27 \%$, and the $\mathrm{CV}$ was $52.73 \mu \mathrm{mol} / \mathrm{g}$, both greater than the maximum limits (Figure 2), indicating the difficulty in evaluating frying oil quality using TPC concentration or $\mathrm{CV}$ alone. After $32 \mathrm{~h}$ of deep-frying, TPC concentration in CO was $29.96 \%$, beyond the maximum Chinese limit (27\%). However, the DG concentration was $4.10 \%$, accounting for $3.34 \%$ of the TPC. Thus, it is problematic to use TPC concentration alone to determine the quality of used CO. TPC concentration is significantly related to TGP [16]. Some European countries use to evaluate frying oil instead of TPC, setting a maximum limit of 10\%-16\% [17]. Using this limit, along with the concentration of TPC (27\%) and TGP (10-16\%), we suggest that the frying life of $\mathrm{CO}$ is $32 \mathrm{~h}$.

\section{Conclusion}

The polar components isolated from used CO possessed pro-oxidant activity. This activity quality affects the storage of refined oil and important in the monitoring of frying oils quality. First, we found polar compounds in commercially refined oil. In order to ensure a long shelf life, it is critical that the refining process decrease the formation of polar compounds. Second, appropriate actions are necessary to decrease the formation of polar compounds during oil frying. We found that excessive accumulation of polar components accelerated the deterioration of the frying oil. Our HPSEC analysis indicated that, because of the high DG content in used CO, the existing standards for used frying oil (maximum 27\% TPC concentration) may be misleading. Thus, we suggest that TPC concentration in combination with another reliable index, TGP (maximum concentration 10-16\%), is a more accurate method by which to determine the frying life of edible oil.

\section{Acknowledgements}

The authors would like to thank the Key Laboratory of Non-wood Forest Breeding and Cultivation of the State Forestry Administration of P R China and Department of Hunan Forestry for their important experiment platform and financial support (No. XLB201618) in the research project.

\section{Statement of Competing Interests}

The authors have no competing interests

\section{List of Abbreviations}

CO Camellia seed oil;

OSI Oxidation induction time

HPSEC High performance size exclusion chromatography;

TPC Total polar compounds;

TGO Triacylglycerol oligomers;

TGD Triacylglycerol dimmers;

ox-TGM oxidized-Triacylglycerol monomers;

DG Diacylglycerols;

FFA Free fatty acid;

\section{References}

[1] Aladedunye, A. F. (2011). Inhibiting thermo-oxidative degradation of oils during frying (Doctoral dissertation, Lethbridge, Alta.: University of Lethbridge, Dept. of Chemistry and Biochemistry, 2011)

[2] Zhang, Q., Saleh, A. S., Chen, J., \& Shen, Q. (2012). Chemical alterations taken place during deep-fat frying based on certain reaction products: a review. Chemistry and physics of lipids, 165: 662-681.

[3] Ahromrit, A., \& Nema, P. K. (2010). Heat and mass transfer in deep-frying of pumpkin, sweet potato and taro. Journal of food science and technology, 47: 632-637. 
[4] Choe, E., \& Min, D. B. (2007). Chemistry of deep - fat frying oils. Journal of food science, 72(5).

[5] Warner, K. (2002). Chemistry of frying oils. FOOD SCIENCE AND TECHNOLOGY-NEW YORK-MARCEL DEKKER-, 205-222.

[6] Farhoosh, R., \& Tavassoli-Kafrani, M. H. (2010). Polar compounds distribution of sunflower oil as affected by unsaponifiable matters of Bene hull oil (BHO) and tertiarybutylhydroquinone (TBHQ) during deep-frying. Food chemistry, 122: 381-385.

[7] Bansal, G., Zhou, W., Barlow, P. J., Lo, H. L., \& Neo, F. L. (2010). Performance of palm olein in repeated deep frying and controlled heating processes. Food chemistry, 121: 338-347.

[8] Kalogianni, E. P., Karastogiannidou, C., \& Karapantsios, T. D. (2009). Effect of the presence and absence of potatoes under repeated frying conditions on the composition of palm oil. Journal of the American Oil Chemists' Society, 86: 561-571.

[9] Gomes, T., Delcuratolo, D., \& Paradiso, V. M. (2008). Prooxidant action of polar triglyceride oligopolymers in edible vegetable oils. European Food Research and Technology, 226: 409-1414.

[10] Xue, B., \& Cao, W. M. (2013). Detection of relative molecular weight of oxidized triglycerides polymers by high performance size-exclusion chromatography. China Oils and Fats, 38: 87-90.

[11] Gomes, T., Delcuratolo, D., Paradiso, V. M., Summo, C., \& Caponio, F. (2011). Pro-oxidant activity of oxidized triacylglycerols in olive oil and comparison with pro-oxidan action of polar triacylglycerol oligopolymers. LWT-Food Science and Technology, 44: 1236-1239.

[12] Li, J. W., Cai, W. C., \& Liu, Y. F. (2015). Effect of the total polar components of frying oil on inhibition and induction of cel. Science and Technology of Food Industry, 36: 358-359.

[13] Ng, C. Y., Leong, X. F., Masbah, N., Adam, S. K., Kamisah, Y., \& Jaarin, K. (2014). Heated vegetable oils and cardiovascular disease risk factors. Vascular pharmacology, 61: 1-9

[14] Venkata, R. P., \& Subramanyam, R. (2016). Evaluation of the deleterious health effects of consumption of repeatedly heated vegetable oil. Toxicology Reports, 3: 636-643.

[15] Dobarganes, M. C., \& Márquez-Ruiz, G. (2007). Formation and analysis of oxidized monomeric, dimeric and higher oligomeric triglycerides. Deep frying: chemistry nutrition and practical applications, 2.

[16] Bastida, S., \& Sánchez-Muniz, F. J. (2002). Polar content vs. TAG oligomer content in the frying-life assessment of monounsaturated and polyunsaturated oils used in deep-frying. Journal of the American Oil Chemists' Society, 79: 447-451.

[17] Firestone, D., \& Erickson, M. D. (2006). Regulation of frying fat and oil. Deep frying: Chemistry, nutrition, and practical applications, (Ed. 2), 373-385.

[18] He, D.Y., Yang, J.F., Zhou, Y.Z., \& Dong, l. (2009). Review on Camellia oleifera. Life Science Research, 13: 459-465.

[19] Guo, L. X., Xu, X. M., Yuan, J. P., Wu, C. F., \& Wang, J. H. (2010). Characterization and authentication of significant Chinese edible oilseed oils by stable carbon isotope analysis. Journal of the American Oil Chemists' Society, 87: 839-848.

[20] Zhu, B., Zhong, H.Y., Cao, Q.M., \& Long, Q.Z. (2010). Advance in research on bioactive compounds in Camellia spp. Non-wood forest research, 28: 140-145.

[21] Zhang, D.S., Jing, Q.Z., Wang, X.G., \& Xue, Y.L. (2013). Research status of nutrition quality of camellia oleifera seed and oil. Science and technology of cereals, oils and foods, 21: 53-56.

[22] Wang, J. Y., \& Zhong, H. Y. (2014). Determination of the content of phenols in Camellia oil by RP-HPSEC with internal standard method. Journal of the chinese cereals and oils association, 29: 107-111.

[23] Wang, J. Y., Zhong, H. Y., Zhu, X. Y., \& Zhou B. (2013). Study on antioxidant activity of polyphenolic compounds from Camellia polydonata seeds. Food and Machinery, 29: 105-107.
[24] Gong, D.P, Chen, Y.L., Zhang, H.M., Hu, Y.C., \& Huang, H (2011). Oxidative stability of purified DHA oil from schizochytrium sp. Food Chemistry, 32: 79-85

[25] Gong, D. P., Hu, Y.C., Zhang, H.M., \& Chen, Y.L. (2010). Effect of free fatty acid on the oxidative stability of DHA oil. Food and Fermentation Industries, 36: 30-33.

[26] Kleiner-Shuhler, L., Vázquez, L., \& Akoh, C. C. (2011). Purification of stearidonic acid from modified soybean oil by argentation silica gel column chromatography. Journal of the American Oil Chemists' Society, 88: 1161-1171.

[27] GB/T 5009.37-2003: Method for analysis of hygienic standard of edible oils. China Standard Press, Beijing.

[28] GB/T 5009.202-2003 : Determination of polar compouds in edible vegetable oils used in frying food. China Standard Press, Beijing.

[29] IUPAC Standard Method 2.507, In: Gold, V.(Ed), Standard methods for the analysis of oils, fats and derivatives, 7th ed. IUPAC press, Blackwell, Oxford 1987.

[30] Márquez-Ruiz, G. (2009). Determination of polar compounds in used frying oils and fats by adsorption chromatography. AOCS lipid library.

[31] Dobarganes, M. C., Velasco, J., \& Dieffenbacher, A. (2000). Determination of polar compounds, polymerized and oxidized triacylglycerols, and diacylglycerols in oils and fats: results of collaborative studies and the standardized method (Technical report). Pure and Applied Chemistry, 72: 1563-1575.

[32] Márquez-Ruiz, G. (2014). Determination of Oxidized Monomeric, Dimeric and Oligomeric Triacylglycerols; Diacylglycerols and Free Fatty Acids.

[33] Kim, H. J., Hahm, T. S., \& Min, D. B. (2007). Hydroperoxide as a prooxidant in the oxidative stability of soybean oil. Journal of the American Oil Chemists' Society, 84: 349-355.

[34] Fan, H. Y., Sharifudin, M. S., Hasmadi, M., \& Chew, H. M. (2013). Frying stability of rice bran oil and palm olein. International Food Research Journal, 20: 403-407.

[35] Aladedunye, F., \& Przybylski, R. (2013). Frying stability of high oleic sunflower oils as affected by composition of tocopherol isomers and linoleic acid content. Food chemistry, 141: 2373-2378.

[36] Erickson, M. D. (Ed.). (2015). Deep frying: chemistry, nutrition, and practical applications. Elsevier.

[37] Kupranycz, D. B., Amer, M. A., \& Baker, B. E. (1986). Effects of thermal oxidation on the constitution of butterfat, butterfat fractions and certain vegetable oils. Journal of the American Oil Chemists' Society, 63: 332-337.

[38] Combe, N., Constantin, M. J., \& Entressangles, B. (1981) Lymphatic absorption of nonvolatile oxidation products of heated oils in the rat. Lipids, 16: 8-14.

[39] Berger, K. G., \& Idris, N. A. (2005). Formulation of zero-trans acid shortenings and margarines and other food fats with products of the oil palm. Journal of the American Oil Chemists' Society, 82: 775-782.

[40] Cao, W.M., Xue, B., Cao, J., Yuan, C., \& Li, Q. Z. (2011). Correlation of Oxidized Triglyceride Polymers to the Degree of Oil Oxidation. Cereals and Oils, 4: 1-5.

[41] Ruiz-Méndez, M. V., Márquez-Ruiz, G., \& Dobarganes, M. C. (1997). Relationships between quality of crude and refined edible oils based on quantitation of minor glyceridic compounds. Food chemistry, 60: 549-554.

[42] Erickson, D. R. (Ed.). (1990). Edible fats and oils processing: basic principles and modern practices: World Conference Proceedings. The American Oil Chemists Society.

[43] Farhoosh, R., \& Pazhouhanmehr, S. (2009). Relative contribution of compositional parameters to the primary and secondary oxidation of canola oil. Food Chemistry, 114: 1002-1006.

[44] Dobarganes, M. C., Pérez - Camino, M. C., \& Márquez - Ruiz, G. (1988). High performance size exclusion chromatography of polar compounds in heated and non - heated fats. European Journal of Lipid Science and Technology, 90: 308-311. 